\title{
$q$-Generalized Linear Operator on Bounded Functions of Complex Order
}

\author{
Rizwan Salim Badar*(D) and Khalida Inayat Noor (D) \\ Department of Mathematics, COMSATS University Islamabad, Islamabad 44000, Pakistan; \\ khalidainayat@comsats.edu.pk \\ * Correspondence: rizwansbadar@gmail.com
}

Received: 16 June 2020; Accepted: 9 July 2020; Published: 14 July 2020

check for updates

\begin{abstract}
This article presents a $q$-generalized linear operator in Geometric Function Theory (GFT) and investigates its application to classes of analytic bounded functions of complex order $S_{q}(c ; M)$ and $C_{q}(c ; M)$ where $0<q<1,0 \neq c \in \mathbb{C}$, and $M>\frac{1}{2}$. Integral inclusion of the classes related to the $q$-Bernardi operator is also proven.
\end{abstract}

Keywords: q-difference operator; subordinating factor sequence; bounded analytic functions of complex order; q-generalized linear operator

MSC: Primary 30C45; Secondary 30C50; $30 H 05$

\section{Introduction}

Quantum calculus or $q$-calculus is attributed to the great mathematicians L.Euler and C. Jacobi, but it became popular when Albert Einstein used it in quantum mechanics in his paper [1] published in 1905. F.H. Jackson $[2,3]$ introduced and studied the $q$-derivative and $q$-integral in a proper way. Later, quantum groups gave the geometrical aspects to $q$-calculus. It is pertinent to mention that $q$-calculus can be considered an extension of classical calculus discovered by I. Newton and G.W. Leibniz. In fact, the operators defined as:

$$
d_{h} f(z)=\frac{f(z+h)-f(z)}{h}
$$

and:

$$
d_{q} f(z)=\frac{f(z)-f(q z)}{(1-q) z}, 0<q<1,
$$

where $z \in \mathbb{C}$ and $h>0$ are the $h$-derivative and $q$-derivative, respectively, where $h$ is Planck's constant, are related as: $q=e^{i h}=e^{2 \pi i \bar{h}}$ where $\bar{h}=h / 2 \pi$. Srivastava [4] applied the concepts of $q$-calculus by using the basic (or $q$-) hypergeometric functions in Geometric Function Theory (GFT). Ismail [5] and Agarwal [6] introduced the class of q-starlike functions by using the $q$-derivative. The $q$-close-to-convex functions were defined in [7], and Sahoo and Sharma [8] obtained several interesting results for $q$-close-to-convex functions. Several convolution and fractional calculus $q$-operators were defined by the researchers, which were reposited by Srivastava in [9]. Darus [10] defined a new differential operator called the $q$-generalized operator by using $q$-hypergeometric functions. Let $A$ be the class of functions of the form:

$$
f(z)=z+\sum_{k=2}^{\infty} a_{k} z^{k}
$$


analytic in the open unit disc $E=\{z:|z|<1\}$.

Let $f(z)$ be given by $(1)$ and $g(z)$ defined as:

$$
g(z)=z+\sum_{k=2}^{\infty} b_{k} z^{k}
$$

The Hadamard product (or convolution) of $f$ and $g$ is defined by:

$$
(f * g)(z)=z+\sum_{k=2}^{\infty} a_{k} b_{k} z^{k}
$$

Let $f, h$ be analytic functions. Then, $f$ is subordinate to $h$, written as $f \prec h$ or $f(z) \prec h(z), z \in E$, if there exists a Schwartz function $\omega(z)$ analytic in $E$ with $\omega(0)=0$ and $|\omega(z)|<1$ for $z \in E$, such that $f(z)=h(\omega(z))$. If $h$ is univalent in $E$, then $f \prec h$, if and only if $f(0)=h(0)$ and $f(E) \subset h(E)$.

A sequence $\left\{b_{k}\right\}_{k=1}^{\infty}$ of complex numbers is a subordinating factor if, whenever $f(z)=$ $\sum_{k=1}^{\infty} a_{k} z^{k}, a_{1}=1$ is regular, univalent, and convex in $E$, we have $\sum_{n=1}^{\infty} b_{n} a_{n} z^{n} \prec f(z), z \in E$ [11].

We recall some basic concepts from $q$-calculus that are used in our discussion and refer to $[2,3,12]$ for more details.

A subset $B \subset \mathbb{C}$ is called $q$-geometric if $z q \in B$ whenever $z \in B$, and it contains all the geometric sequences $\left\{z q^{k}\right\}_{0}^{\infty}$. In GFT, the $q$-derivative of $f(z)$ is defined as:

$$
d_{q} f(z)=\frac{f(z)-f(q z)}{(1-q) z}, q \in(0,1), \quad(z \in B \backslash\{0\}),
$$

and $d_{q} f(0)=f^{\prime}(0)$. For a function $g(z)=z^{k}$, the $q$-derivative is:

$$
d_{q} g(z)=[k] z^{k-1}
$$

where $[k]=\frac{1-q^{k}}{1-q}=1+q+q^{2}+\ldots .+q^{k-1}$.

We note that as $q \rightarrow 1^{-}, d_{q} f(z) \rightarrow f^{\prime}(z)$, which is the ordinary derivative. From (1), we deduce that:

$$
d_{q} f(z)=1+\sum_{k=2}^{\infty}[k] a_{k} z^{k} .
$$

Let $f(z)$ and $g(z)$ be defined on a $q$-geometric set $B$. Then, for complex numbers $a, b$, we have:

$$
\begin{gathered}
d_{q}(a f(z) \pm b g(z))=a d_{q} f(z) \pm b d_{q} g(z) . \\
d_{q}(f(z) g(z))=f(q z) d_{q} g(z)+g(z) d_{q} f(z) . \\
d_{q}\left(\frac{f(z)}{g(z)}\right)=\frac{g(z) d_{q} f(z)-f(z) d_{q} g(z)}{g(z) g(q z)}, g(z) g(q z) \neq 0 . \\
d_{q}(\log f(z))=\frac{\ln q^{-1}}{1-q} \frac{d_{q} f(z)}{f(z)} .
\end{gathered}
$$

Jackson [2] introduced the q-integral of a function $f$, given by:

$$
\int_{0}^{z} f(t) d_{q} t=z(1-q) \sum_{k=0}^{\infty} q^{k} f\left(q^{k} z\right),
$$

provided that the series converges.

For any non-negative integer $n$, the $q$-number shift factorial is defined as:

$$
[n] !=\left\{\begin{array}{cc}
{[1][2] \ldots[n]} & \text { if } n \neq 0, \\
1 & \text { if } n=0
\end{array}\right.
$$


Let $\lambda \in \mathbb{R}$ and $n \in \mathbb{N}$; the $q$-generalized Pochhammer symbol is defined as:

$$
[\lambda]_{n}=[\lambda][\lambda+1][\lambda+2] \ldots .[\lambda+n-1] .
$$

The $q$-Gamma function is defined for $\lambda>0$ as:

$$
\Gamma_{q}(\lambda+1)=[\lambda] \Gamma_{q}(\lambda) \quad \text { and } \quad \Gamma_{q}(1)=1 .
$$

For complex parameters $a_{i}(1 \leq i \leq l), b_{j} \neq 0,-1,-2, \ldots(1 \leq j \leq m)$ with $l \leq m+1$, the basic $q$-hypergeometric function is defined as,

$$
{ }_{l} F_{m}\left(a_{1}, \ldots a_{l} ; b_{1}, \ldots, b_{m}, z\right)=\sum_{k=0}^{\infty} \frac{\left(a_{1}\right)_{k} \ldots\left(a_{l}\right)_{k}}{(q)_{k}\left(b_{1}\right)_{k} \ldots\left(b_{m}\right)_{k}}\left[(-1)^{n} q^{\left(\begin{array}{c}
n \\
2
\end{array}\right)}\right]^{1+m-l} z^{k}
$$

with $\left(\begin{array}{l}n \\ 2\end{array}\right)=\frac{n(n-1)}{2}$ and $l, m \in \mathbb{N}_{0}=\mathbb{N} \cup\{0\}$. Here, the $q$-shifted factorial is defined for $a \in \mathbb{C}$ as:

$$
(a)_{k}=\left\{\begin{array}{cl}
(1-a)(1-a q) \ldots\left(1-a q^{k-1}\right) & \text { if } k \in \mathbb{N}, \\
1 & \text { if } k=0 .
\end{array}\right.
$$

Let $l=m+1, a_{1}=q^{\lambda+1}(\lambda>-1), a_{i}=q(\forall 2 \leq i \leq l)$, and $b_{j}=q(\forall 1 \leq j \leq m)$, and by using the property $\left(q^{a}\right)_{k}=\Gamma_{q}(a+k)(1-q)^{k} / \Gamma_{q}(a)$, from (2), we get the function,

$$
F_{q, \lambda+1}(z)=z+\sum_{k=2}^{\infty} \frac{\Gamma_{q}(\lambda+k)}{[k-1] ! \Gamma_{q}(\lambda+1)} z^{k}=z+\sum_{k=2}^{\infty} \frac{[\lambda+1]_{k-1}}{[k-1] !} z^{k}, z \in E .
$$

In [13], the $q$-Srivastava-Attiya convolution operator is defined as:

$$
G_{q, a}^{s}(z)=z+\sum_{k=2}^{\infty}\left(\frac{[1+a]}{[k+a]}\right)^{s} z^{k}, z \in E,
$$

$\left(a \in \mathbb{C} \backslash \mathbb{Z}_{0}^{-} ; s \in \mathbb{C}\right.$ when $|z|<1 ; \operatorname{Re}(s)>1$ when $\left.|z|=1\right)$.

Using convolution, the operator $D_{q, a, \lambda}^{s}$ for $\lambda>-1$ is defined as:

$$
\begin{aligned}
D_{q, a, \lambda}^{s} f(z) & =J_{q, a, \lambda}^{s}(z) * f(z) \\
& =z+\sum_{k=2}^{\infty}\left(\frac{[k+a]}{[1+a]}\right)^{s} \frac{[\lambda+1]_{k-1}}{[k-1] !} a_{k} z^{k}, z \in E,
\end{aligned}
$$

where:

$$
J_{q, a, \lambda}^{s}(z)=\left(G_{q, a}^{s}(z)\right)^{-1} * F_{q, \lambda+1}(z)=z+\sum_{k=2}^{\infty}\left(\frac{[k+a]}{[1+a]}\right)^{s} \frac{[\lambda+1]_{k-1}}{[k-1] !} z^{k} .
$$

It is a convergent series with a radius of convergence of one. We observe that $D_{q, a, 0}^{0} f(z)=f(z)$ and $D_{q, 0,0}^{1} f(z)=z d_{q} f(z)$. The operator $D_{q, a, \lambda}^{s}$ reduces to known linear operators for different values of parameters $a, s$, and $\lambda$ as:

(i) If $q \rightarrow 1^{-}$, it reduces to the operator $D_{a, \lambda}^{s}$ discussed by Noor et al. in [14].

(ii) For $s=0$, it is a $q$-Ruscheweyh differential operator [15].

(iii) If $s=-1, \lambda=0$, and $q \rightarrow 1^{-}$, it is an Owa-Srivastava integral operator [16].

(iv) If $s \in \mathbb{N}_{0}, a=1, \lambda=0$, and $q \rightarrow 1^{-}$, it reduces to the generalized Srivastava-Attiya integral operator [17].

(v) If $s \in \mathbb{N}_{0}, a=0, \lambda=0$, it is a $q$-Salagean differential operator [18].

(vi) For $s, \lambda \in \mathbb{N}_{0}$, and $a=0$, it is the operator defined in [19]. 
The following identities hold for the operator $D_{q, a, \lambda}^{s} f(z)$,

$$
\begin{aligned}
z d_{q}\left(D_{q, a, \lambda}^{s} f(z)\right) & =\left(\frac{[1+a]}{q^{a}}\right) D_{q, a, \lambda}^{s+1} f(z)-\frac{[a]}{q^{a}} D_{q, a, \lambda}^{s} f(z) \\
z d_{q}\left(D_{q, a, \lambda}^{s} f(z)\right) & =\left(\frac{[1+\lambda]}{q^{\lambda}}\right) D_{q, a, \lambda+1}^{s} f(z)-\frac{[\lambda]}{q^{\lambda}} D_{q, a, \lambda}^{s} f(z) .
\end{aligned}
$$

Let $P(q)$ be the class of functions of the form $p(z)=1+c_{1} z+c_{2} z^{2}+\ldots$. , analytic in $E$, and satisfying:

$$
\left|p(z)-\frac{1}{1-q}\right| \leq \frac{1}{1-q}, \quad(z \in E, q \in(0,1)) .
$$
$z \in E$.

It is known from [20] that $p \in P(q)$ implies $p(z) \prec \frac{1+z}{1-q z}$. It follows immediately that $\operatorname{Re} p(z)>0$,

The classes of bounded $q$-starlike functions $S_{q}(c, M)$ and bounded $q$-convex functions $C_{q}(c, M)$ of complex order $c$ were defined in [21], respectively, as:

$$
\begin{gathered}
S_{q}(c, M)=\left\{f \in A:\left|\frac{c-1+\frac{z d_{q} f(z)}{f(z)}}{c}-M\right|<M\right\}, \\
\left(c \in \mathbb{C}^{*} ; M>\frac{1}{2}, z \in E\right),
\end{gathered}
$$

or equivalently,

$$
\begin{gathered}
S_{q}(c, M)=\left\{f \in A: \frac{z d_{q} f(z)}{f(z)} \prec \frac{1+\{c(1+m)-m\} z}{1-m z}\right\}, \\
\left(c \in \mathbb{C}^{*} ; m=1-\frac{1}{M} ; M>\frac{1}{2}\right) .
\end{gathered}
$$

The class of bounded $q$-convex functions $C_{q}(c, M)$ of complex order $c$ is defined as:

$$
\begin{gathered}
C_{q}(c, M)=\left\{f \in A: \mid \frac{\left.c-1+\frac{d_{q}\left(z d_{q} f(z)\right)}{d_{q} f(z)}-M \mid<M\right\},}{c}-\right. \\
\left(c \in \mathbb{C}^{*} ; M>\frac{1}{2}, z \in E\right),
\end{gathered}
$$

or equivalently,

$$
\begin{gathered}
C_{q}(c, M)=\left\{f \in A: \frac{d_{q}\left(z d_{q} f(z)\right)}{d_{q} f(z)} \prec \frac{1+\{c(1+m)-m\} z}{1-m z}\right\} \\
\left(c \in^{*} ; m=1-\frac{1}{M} ; M>\frac{1}{2}\right) .
\end{gathered}
$$

Using the operator $D_{q, a, \lambda}^{s} f(z)$, we now define the following new classes $S_{q, a, s, \lambda}(c, M)$ and $C_{q, a, s, \lambda}(c, M)$ as: 


$$
\begin{aligned}
S_{q, a, s, \lambda}(c, M) & =\left\{f \in A: \frac{z\left(d_{q} D_{q, a, \lambda}^{s}(f(z))\right)}{D_{q, a, \lambda}^{s}(f(z))} \prec \frac{1+\{c(1+m)-m\} z}{1-m z}, z \in E\right\}, \\
& \left(0<q<1, c \in^{*} ; m=1-\frac{1}{M} ; M>\frac{1}{2}\right) .
\end{aligned}
$$

Special cases:

(i) If $c=1, m=1$, and $q \rightarrow 1^{-}$, then $S_{q, a, s, \lambda}(c, M)$ reduces to class $S^{s}(a, \lambda)$ discussed in [22].

(ii) If $c=1, s=0, \lambda=0, m=-q$, then $S_{q, a, s, \lambda}(c, M)$ reduces to class $S_{q}^{*}$ introduced by Noor et al. [23].

(iii) If $s=0, c=\frac{m}{1+m}(-1<m<0), m=-q$, then $S_{q, a, s, \lambda}(c, M)$ reduces to class $S T_{q}$ studied by Noor [24].

(iv) If $s=0, \lambda=0, c=a e^{-i \beta} \cos \beta\left(a \in \mathbb{C}^{*},|\beta|<\frac{\pi}{2}\right)$, and $q \rightarrow 1^{-}$, then $S_{q, a, s, \lambda}(c, M)$ becomes special cases of Janowski $\beta$-spiral like functions of complex order $S^{\beta}(A, B, a)$ discussed in [25].

(v) If $s \in \mathbb{N}_{0}, \lambda=0, a=0$, and $q \rightarrow 1^{-}$, then $S_{q, a, s, \lambda}(c, M)$ reduces to class $H_{n}(c, M)$ discussed by Aouf et al. in [26].

(vi) If $0<c \leq 1,-1<m<0$, and $q \rightarrow 1^{-}$, then $S_{q, a, s, \lambda}(c, M)$ becomes a special case of the class $S_{a, \lambda}^{s}(\eta$, $A, B)$ with $\eta=0$ discussed in [19].

A function $f \in A$ is in the class $S_{q, a, s, \lambda}(c, M)$ if and only if:

$$
\left|\frac{\frac{z d_{q}\left(D_{q, a, \lambda}^{s} f(z)\right)}{D_{q, a, \lambda}^{s} f(z)}-1}{A-B\left\{\frac{z d_{q}\left(D_{q, a, \lambda}^{s} f(z)\right)}{D_{q, a, \lambda}^{s} f(z)}\right\}}\right|<1,
$$

where $A=c(1+m)-m$ and $B=-m$.

The class $C_{q, a, s, \lambda}(c, M)$ is defined as:

$$
\begin{aligned}
C_{q, a, s, \lambda}(c, M)= & \left\{f \in A: \frac{d_{q}\left(z d_{q}\left(D_{q, a, \lambda}^{s} f(z)\right)\right.}{d_{q}\left(D_{q, a, \lambda}^{s} f(z)\right)} \prec \frac{1+\{c(1+m)-m\} z}{1-m z}, z \in E\right\}, \\
& \left(0<q<1, c \in \mathbb{C}^{*} ; m=1-\frac{1}{M} ; M>\frac{1}{2}\right) .
\end{aligned}
$$

It is easy to see that $f \in C_{q, a, s, \lambda}(c, M) \Leftrightarrow z d_{q} f \in S_{q, a, s, \lambda}(c, M)$. In order to develop results for the classes $S_{q, a, s, \lambda}(c, M)$ and $C_{q, a, s, \lambda}(c, M)$, we need the following:

Lemma 1 ([27]). Let $\beta$ and $\gamma$ be complex numbers with $\beta \neq 0$, and let $h(z)$ be regular in $E$ with $h(0)=1$ and $\operatorname{Re}[\beta h(z)+\gamma]>0$. If $p(z)=1+p_{1} z+p_{2} z^{2}+\ldots$ is analytic in $E$, then $p(z)+\frac{z d_{q} p(z)}{\beta p(z)+\gamma} \prec h(z) \Rightarrow p(z) \prec$ $h(z)$.

Lemma 2 ([11]). The sequence $\left\{b_{n}\right\}_{n=1}^{\infty}$ is a subordinating factor sequence if and only if:

$$
\operatorname{Re}\left\{1+2 \sum_{k=1}^{\infty} b_{k} z^{k}\right\}>0, z \in E .
$$

2. Properties of Classes $S_{q, a, s, \lambda}(c, M)$ and $C_{q, a, s, \lambda}(c, M)$

We start the section with the necessary and sufficient condition for a function to be in the class $S_{q, a, s, \lambda}(c, M)$. 
Theorem 1. Let $f \in A$. Then, $f \in S_{q, a, s, \lambda}(c, M)$ if and only if:

$$
\sum_{k=2}^{\infty}\{[k]-1+|c(1+m)+m([k]-1)|\} \frac{[\lambda+1]_{k-1}}{[k-1] !}\left|\left(\frac{[k+a]}{[1+a]}\right)^{s}\right|\left|a_{k}\right|<|c(1+m)|,
$$

where $m=1-\frac{1}{M},\left(M>\frac{1}{2}\right)$.

Proof. Let us assume first that Inequality (6) holds. To show $f \in S_{q, a, s, \lambda}(c, M)$, we need to prove Inequality (5).

$$
\begin{aligned}
\left|\frac{\frac{z\left(d_{q}\left(D_{q, a, \lambda}^{s} f(z)\right)\right.}{D_{q, a, \lambda}^{s} f(z)}-1}{A-B\left\{\frac{z d_{q}\left(D_{q, a, \lambda}^{s} f(z)\right)}{D_{q, a, \lambda}^{s} f(z)}\right\} \mid}\right| & \left|\frac{\sum_{k=2}^{\infty}\left(\frac{[k+a]}{[1+a]}\right)^{S} \cdot \frac{[\lambda+1]_{k-1}}{[k-1] !}([k]-1) a_{k} z^{k}}{(A-B) z+\sum_{k=2}^{\infty}(A-B[k])\left(\frac{[1+a]}{[k+a]}\right)^{S} \cdot \frac{[\lambda+1]_{k-1}}{[k-1] !} a_{k} z^{k}}\right| \\
& \leq \frac{\sum_{k=2}^{\infty}\left|\left(\frac{[k+a]}{[1+a]}\right)^{s}\right| \cdot \frac{[\lambda+1]_{k-1}}{[k-1] !}([k]-1)\left|a_{k}\right|}{|A-B|-\left|\sum_{k=2}^{\infty}(A-B[k])\left(\frac{[k+a]}{[1+a]}\right)^{S} \cdot \frac{[\lambda+1]_{k-1}}{[k-1] !} a_{k}\right|} \\
& \leq \frac{\sum_{k=2}^{\infty}\left|\left(\frac{[k+a]}{[1+a]}\right)^{s}\right| \cdot \frac{[\lambda+1]_{k-1}}{[k-1] !}([k]-1)\left|a_{k}\right|}{|c(1+m)|-\sum_{k=2}^{\infty}|c(1+m)+m([k]-1)| \frac{[\lambda+1]_{k-1}}{[k-1] !}\left|\left(\frac{[k+a]}{[1+a]}\right)^{s}\right|\left|a_{k}\right|} \\
& <1 .
\end{aligned}
$$

Hence, $f \in S_{q, a, s, \lambda}(c, M)$ by using Inequality (6). Conversely, let $f \in S_{q, a, s, \lambda}(c, M)$ be of the form (1), then:

$$
\left|\frac{\frac{z\left(d_{q}\left(D_{q, a, \lambda}^{s} f(z)\right)\right.}{D_{q, a, \lambda}^{s}(f(z))}-1}{A-B\left\{\frac{z d_{q}\left(D_{q, a, \lambda}^{s} f(z)\right)}{D_{q, a, \lambda}^{s} f(z)}\right\}}\right|=\left|\frac{\sum_{k=2}^{\infty}\left(\frac{[k+a]}{[1+a]}\right)^{S} \cdot \frac{[\lambda+1]_{k-1}}{[k-1] !}([k]-1) a_{k} z^{k}}{(A-B) z+\sum_{k=2}^{\infty}(A-B[k])\left(\frac{[k+a]}{[1+a]}\right)^{S} \cdot \frac{[\lambda+1]_{k-1}}{[k-1] !} a_{k} z^{k}}\right| .
$$

Since $|\operatorname{Re} z| \leq|z|$, we have:

$$
\operatorname{Re}\left\{\left|\frac{\sum_{k=2}^{\infty}\left(\frac{[k+a]}{[1+a]}\right)^{S} \cdot \frac{[\lambda+1]_{k-1}}{[k-1] !}([k]-1) a_{k} z^{k}}{(A-B) z+\sum_{k=2}^{\infty}(A-B[k])\left(\frac{[k+a]}{[1+a]}\right)^{S} \cdot \frac{[\lambda+1]_{k-1}}{[k-1] !} a_{k} z^{k}}\right|\right\}<1
$$

Now, we choose values of $z$ on the real axis such that $z d_{q}\left(D_{q, a, \lambda}^{s} f(z)\right) / D_{q, a, \lambda}^{s} f(z)$ is real. Letting $z \rightarrow 1^{-}$through real values, after some calculations, we obtain Inequality (6).

Remark 1. (i) If $q \rightarrow 1^{-}, s \in \mathbb{N}_{0}, a=0$, and $\lambda=0$, the above result reduces to the sufficient condition for $f(z)$ to be in class $H_{n}(c, M)\left(c \in \mathbb{C}^{*}, M>\frac{1}{2}\right)$ discussed in [26]. (ii) If $c=1-\alpha(\alpha \in[0,1)), m=0, \lambda=0$, and $q \rightarrow 1^{-}$, the above result reduces to the sufficient condition for $f(z)$ to be in class $S_{s, a}^{*}(\alpha)$ discussed in [28].

Theorem 2. Let $f_{i} \in S_{q, a, s, \lambda}(c, M)$ having the form:

$$
f_{i}(z)=z+\sum_{k=2}^{\infty} a_{k, i} z^{k}, \quad \text { for } i=1,2,3, . ., l .
$$

Then, $F \in S_{q, a, s, \lambda}(c, M)$, where $F(z)=\sum_{i=1}^{l} c_{i} f_{i}(z)$ with $\sum_{i=1}^{ł} c_{i}=1$.

Proof. From Theorem 1, we can write: 


$$
\sum_{k=2}^{\infty}\left\{\frac{\{[k]-1+|b(1+m)+m([k]-1)|\} \frac{[\lambda+1]_{k-1}}{[k-1] !}\left|\left(\frac{[k+a]}{[1+a]}\right)^{s}\right|}{|b(1+m)|}\right\} a_{k, i}<1 .
$$

Therefore:

$$
\begin{aligned}
F(z) & =\sum_{i=1}^{l} c_{i}\left(z+\sum_{k=2}^{\infty} a_{k, i} z^{k}\right) \\
& =z+\sum_{k=2}^{\infty}\left(\sum_{i=1}^{l} c_{i} a_{k, i}\right) z^{k}
\end{aligned}
$$

where however due to (7), we have:

$$
\begin{aligned}
& \sum_{k=2}^{\infty} \frac{\{[k]-1+|b(1+m)+m([k]-1)|\} \frac{[\lambda+1]_{k-1}}{[k-1] !}\left|\left(\frac{[k+a]}{[1+a]}\right)^{s}\right|}{|b(1+m)|}\left(\sum_{i=2}^{l} c_{i} a_{k, i}\right) \\
& =\sum_{i=2}^{l}\left[\frac{\{[k]-1+|b(1+m)+m([k]-1)|\} \frac{[\lambda+1]_{k-1}\left|\left(\frac{[k+a]}{[k-1] !}\right)^{s}\right|}{[1+a]} \mid}{|b(1+m)|}\right] c_{i} \leq 1
\end{aligned}
$$

Therefore, $F \in S_{q, a, s, \lambda}(c, M)$.

Theorem 3. Let $f_{i}$ with $i=1,2, \ldots, v$ belong to the class $S_{q, a, s, \lambda}(c, M)$. The arithmetic mean $h$ of $f_{i}$ is given by:

$$
h(z)=\frac{1}{v} \sum_{i=1}^{v} f_{i}(z)
$$

belonging to class $S_{q, a, s, \lambda}(c, M)$.

Proof. From (8), we can write:

$$
h(z)=\frac{1}{v} \sum_{i=1}^{v}\left(z+\sum_{k=2}^{\infty} a_{k, i} z^{k}\right)=z+\sum_{k=2}^{\infty}\left(\frac{1}{v} \sum_{i=1}^{v} a_{k, i}\right) z^{k} .
$$

Since $f_{i} \in S_{q, a, s, \lambda}(c, M)$ for every $i=1,2, \ldots, v$, using (6) and (9), we have:

$$
\begin{aligned}
& \sum_{k=2}^{\infty}\{[k]-1+|b(1+m)+m([k]-1)|\} \frac{[\lambda+1]_{k-1}}{[k-1] !}\left|\left(\frac{[k+a]}{[1+a]}\right)^{s}\right|\left(\frac{1}{v} \sum_{i=1}^{v} a_{k, i}\right) \\
& =\frac{1}{v} \sum_{i=1}^{v}\left(\sum_{k=2}^{\infty}\{[k]-1+|b(1+m)+m([k]-1)|\} \frac{[\lambda+1]_{k-1}}{[k-1] !}\left|\left(\frac{[k+a]}{[1+a]}\right)^{s}\right| a_{k, i}\right) \\
& \leq \frac{1}{v} \sum_{i=1}^{v}(|b(1+m)|)=|b(1+m)|,
\end{aligned}
$$

and this completes the proof.

Now, we give the subordination relation for the functions in class $S_{q, a, s, \lambda}(c, M)$ by using the subordination theorem.

Theorem 4. Let $m=1-\frac{1}{M}\left(M>\frac{1}{2}\right)$. Furthermore, $c \neq 0$ with $\operatorname{Re}(c)>\frac{-m}{2(1+m)}$ when $m>0$ and $\operatorname{Re}(c)<\frac{-m}{2(1+m)}$ when $m<0$ and $\lambda \geq 0$. If $f \in S_{q, a, s, \lambda}(c, M)$, then: 


$$
\frac{\{q+|c(1+m)+m q|\} C_{\lambda, 2} B_{s, a}(2)}{2\left[\{q+|c(1+m)+m q|\} C_{\lambda, 2} B_{s, a}(2)+|c(1+m)|\right]}(f * g)(z) \prec g(z)
$$

where $g(z)$ is a convex function in $E, C_{\lambda, k}=\frac{[\lambda+1]_{k-1}}{[k-1] !}, B_{s, a}(k)=\left|\left(\frac{[k+a]}{[1+a]}\right)^{s}\right|$, and:

$$
\operatorname{Re} f(z)>-1-\frac{(1+m)|c|}{\{q+|c(1+m)+m q|\} C_{\lambda, 2} B_{s, a}(2)} .
$$

The constant $\frac{\{q+|c(1+m)+m q|\} C_{\lambda, 2} B_{s, a}(2)}{2\left[\{q+|c(1+m)+m q|\} C_{\lambda, 2} B_{s, a}(2)+|c(1+m)|\right]}$ is the best estimate.

Proof. Let $f(z) \in S_{q, a, s, \lambda}(c, M)$ and $g(z)=z+\sum_{k=2}^{\infty} c_{k} z^{k}$. Then:

$$
\begin{aligned}
& \frac{\{q+|c(1+m)+m q|\} C_{\lambda, 2} B_{s, a}(2)}{2\left[\{q+|c(1+m)+m q|\} C_{\lambda, 2} B_{s, a}(2)+|c(1+m)|\right]}(f * g)(z) \\
& =\frac{\{q+|c(1+m)+m q|\} C_{\lambda, 2} B_{s, a}(2)}{2\left[\{q+|c(1+m)+m q|\} C_{\lambda, 2} B_{s, a}(2)+|c(1+m)|\right]}\left(z+\sum_{k=2}^{\infty} a_{k} c_{k} z^{k}\right) .
\end{aligned}
$$

Thus, (10) holds true if:

$$
\left\{\frac{\{q+|c(1+m)+m q|\} C_{\lambda, 2} B_{s, a}(2)}{2\left[\{q+|c(1+m)+m q|\} C_{\lambda, 2} B_{s, a}(2)+|c(1+m)|\right]} a_{k}\right\}_{k=1}^{\infty}
$$

is a subordinating factor sequence with $a_{1}=1$. From Lemma 2, it suffices to show:

$$
\operatorname{Re}\left\{1+\sum_{k=1}^{\infty} \frac{\{q+|c(1+m)+m q|\} C_{\lambda, 2} B_{s, a}(2)}{\left[\{q+|c(1+m)+m q|\} C_{\lambda, 2} B_{s, a}(2)+|c(1+m)|\right]} a_{k} z^{k}\right\}>0 .
$$

Now, as $\{[k]-1+|c(1+m)+m([k]-1)|\} C_{\lambda, k} B_{s, a}(k)$ is an increasing function of $k(k \geq 2)$, we have:

$$
\begin{aligned}
& \operatorname{Re}\left\{1+\sum_{k=1}^{\infty} \frac{\{q+|c(1+m)+m q|\} C_{\lambda, 2} B_{s, a}(2)}{\left[\{q+|c(1+m)+m q|\} C_{\lambda, 2} B_{s, a}(2)+|c(1+m)|\right]} a_{k} z^{k}\right\} \\
& =\operatorname{Re}\left\{1+\frac{\{q+|c(1+m)+m q|\} C_{\lambda, 2} B_{s, a}(2)}{\left[\{q+|c(1+m)+m q|\} C_{\lambda, 2} B_{s, a}(2)+|c(1+m)|\right]} z+\right. \\
& \left.+\frac{\sum_{k=2}^{\infty}\{q+|c(1+m)+m q|\} C_{\lambda, 2} B_{s, a}(2) a_{k} z^{k}}{\left[\{q+|c(1+m)+m q|\} C_{\lambda, 2} B_{s, a}(2)+|c(1+m)|\right]}\right\} \\
& \geq 1-\frac{\{q+|c(1+m)+m q|\} C_{\lambda, 2} B_{s, a}(2)}{\left[\{q+|c(1+m)+m q|\} C_{\lambda, 2} B_{s, a}(2)+|c(1+m)|\right]} r- \\
& \frac{\sum_{k=2}^{\infty}\{q+|c(1+m)+m q|\} C_{\lambda, 2} B_{s, a}(2)\left|a_{k}\right| r^{k}}{\left[\{q+|c(1+m)+m q|\} C_{\lambda, 2} B_{s, a}(2)+|c(1+m)|\right]} \\
& >1-\frac{\{q+|c(1+m)+m q|\} C_{\lambda, 2} B_{s, a}(2)}{\left[\{q+|c(1+m)+m q|\} C_{\lambda, 2} B_{s, a}(2)+|c(1+m)|\right]} r- \\
& \frac{(1+m)|c|}{\left[\{q+|c(1+m)+m q|\} C_{\lambda, 2} B_{s, a}(2)+|c(1+m)|\right]} r \\
& >0 . \quad(|z|=r<1)
\end{aligned}
$$

Hence, (14) holds true in $E$, and the subordination result (10) is affirmed by Theorem 4 . The inequality (11) follows by taking $g(z)=\frac{z}{1-z}=\sum_{k=1}^{\infty} z^{k}$ in (10). 
Let us consider the function:

$$
\phi(z)=z-\frac{|c(1+m)|}{\left[\{q+|c(1+m)+m q|\} C_{\lambda, 2} B_{s, a}(2)+|c(1+m)|\right]} z^{2}(z \in E)
$$

which is a member of $S_{q, a, s, \lambda}(c, M)$. Then. by using (10), we have:

$$
\frac{\{q+|c(1+m)+m q|\} C_{\lambda, 2} B_{s, a}(2)}{2\left[\{q+|c(1+m)+m q|\} C_{\lambda, 2} B_{s, a}(2)+|c(1+m)|\right]} \phi(z) \prec \frac{z}{1-z} .
$$

It is easily verified that:

$$
\min \operatorname{Re}\left\{\frac{\{q+|c(1+m)+m q|\} C_{\lambda, 2} B_{s, a}(2)}{2\left[\{q+|c(1+m)+m q|\} C_{\lambda, 2} B_{s, a}(2)+|c(1+m)|\right]} \phi(z)\right\}=-\frac{1}{2} \quad(z \in E),
$$

then the constant $\frac{\{q+|c(1+m)+m q|\} C_{\lambda, 2} B_{s, a}(2)}{2\left[\{q+|c(1+m)+m q|\} C_{\lambda, 2} B_{s, a}(2)+|c(1+m)|\right]}$ cannot be replaced by a larger one.

Remark 2. If $s \in \mathbb{N}_{0}, a=0, \lambda=0$, and $q \rightarrow 1^{-}$, Theorem 4 reduces to the subordination result proven in [29].

Now, we discuss the inclusion results pertaining to classes $S_{q, a, s, \lambda}(c, M)$ and $C_{q, a, s, \lambda}(c, M)$ in reference to parameters $s$ and $\lambda$.

Theorem 5. For any complex number $s, S_{q, a, s+1, \lambda}(c, M) \subset S_{q, a, s, \lambda}(c, M)$ if $\operatorname{Re}\left(\frac{1+\{c(1+m)-m\} z}{1-m z}\right)>$ $\frac{1}{q^{a_{1}}(1-q)}\left\{1-\cos \left(a_{2} \ln q\right)\right\}$ where $a=a_{1}+i a_{2}$.

Proof. Let $f \in S_{q, a, s+1, \lambda}(c, M)$, then:

$$
\frac{z d_{q}\left(D_{q, a, \lambda}^{s+1} f(z)\right)}{D_{q, a, \lambda}^{s+1} f(z)} \prec \frac{1+\{c(1+m)-m\} z}{1-m z},
$$

Let:

$$
h(z)=\frac{1+\{c(1+m)-m\} z}{1-m z}
$$

and:

$$
r(z)=\frac{z d_{q}\left(D_{q, a, \lambda}^{s} f(z)\right)}{D_{q, a, \lambda}^{s} f(z)} .
$$

We will show:

$$
r(z) \prec h(z),
$$

which would prove $S_{q, a, s, \lambda}(c, M) \subset S_{q, a, s+1, \lambda}(c, M)$. From the identity relation (3), after a few calculations, we have:

$$
\frac{z d_{q}\left(D_{q, a, \lambda}^{s} f(z)\right)}{D_{q, a, \lambda}^{s} f(z)}=\frac{[1+a]}{q^{a}} \cdot \frac{D_{q, a, \lambda}^{s+1} f(z)}{D_{q, a, \lambda}^{s} f(z)}-\frac{[a]}{q^{a}} .
$$

After some calculations, we have: 


$$
\begin{aligned}
\frac{D_{q, a, \lambda}^{s+1} f(z)}{D_{q, a, \lambda}^{s} f(z)} & =\frac{1}{[1+a]}\left\{\frac{q^{a} z d_{q}\left(D_{q, a, \lambda}^{s} f(z)\right)}{D_{q, a, \lambda}^{s} f(z)}+[a]\right\} \\
& =\frac{1}{[1+a]}\left\{q^{a} r(z)+[a]\right\} .
\end{aligned}
$$

Applying logarithmic $q$-differentiation, we have:

$$
\frac{z d_{q}\left(D_{q, a, \lambda}^{s+1} f(z)\right)}{D_{q, a, \lambda}^{s+1} f(z)}=r(z)+\frac{z d_{q} r(z)}{r(z)+q^{-a}[a]} .
$$

From (15) and (16), we have:

$$
r(z)+\frac{z\left[d_{q} r(z)\right]}{r(z)+q^{-a}[a]} \prec \frac{1+\{c(1+m)-m\} z}{1-m z} .
$$

If $\operatorname{Re}(h(z))>\frac{1}{q^{a_{1}}(1-q)}\left\{1-\cos \left(a_{2} \ln q\right)\right\}$, then from Lemma 1, it implies:

$$
r(z) \prec h(z),
$$

which implies $f(z) \in S_{q, a, s, \lambda}(c, M)$. Therefore, $S_{q, a, s, \lambda}(c, M) \subset S_{q, a, s+1, \lambda}(c, M)$.

Theorem 6. For any complex number s, $C_{q, a, s+1, \lambda}(c, M) \subset C_{q, a, s, \lambda}(c, M)$ if $\operatorname{Re}\left(\frac{1+\{c(1+m)-m\} z}{1-m z}\right)>$ $\frac{1}{q^{a_{1}}(1-q)}\left\{1-\cos \left(a_{2} \ln q\right)\right\}$ where $a=a_{1}+i a_{2}$.

Proof. It is obvious from the fact $f \in C_{q, a, s, \lambda}(c, M) \Leftrightarrow z d_{q} f \in S_{q, a, s, \lambda}(c, M)$.

Theorem 7. For any complex number $s, S_{q, a, s, \lambda+1}(c, M) \subset S_{q, a, s, \lambda}(c, M)$ if $\operatorname{Re}\left(\frac{1+\{c(1+m)-m\} z}{1-m z}\right)>\frac{1-q^{-\lambda}}{1-q}$, $\lambda>-1$.

Proof. Let $f \in S_{q, a, s, \lambda+1}(c, M)$, then:

$$
\frac{z d_{q}\left(D_{q, a, \lambda+1}^{s} f(z)\right)}{D_{q, a, \lambda+1}^{s} f(z)} \prec \frac{1+\{c(1+m)-m\} z}{1-m z} .
$$

Consider:

$$
h(z)=\frac{1+\{c(1+m)-m\} z}{1-m z}
$$

and:

$$
q(z)=\frac{z d_{q}\left(D_{q, a, \lambda}^{s} f(z)\right)}{D_{q, a, \lambda}^{s} f(z)} .
$$

We will show:

$$
q(z) \prec h(z),
$$

which would conveniently prove $S_{q, a, s, \lambda+1}(c, M) \subset S_{q, a, s, \lambda}(c, M)$. From the identity relation (4), after a few calculations, we have:

$$
\frac{z d_{q}\left(D_{q, a, \lambda}^{s} f(z)\right)}{D_{q, a, \lambda}^{s} f(z)}=\frac{[1+\lambda]}{q^{\lambda}} \frac{D_{q, a, \lambda+1}^{s} f(z)}{D_{q, a, \lambda}^{s} f(z)}-\frac{[\lambda]}{q^{\lambda}} .
$$


After some calculations, we have:

$$
\begin{aligned}
\frac{D_{q, a, \lambda+1}^{s} f(z)}{D_{q, a, \lambda}^{s} f(z)} & =\frac{1}{[1+\lambda]}\left\{\frac{q^{a} \cdot z d_{q}\left(D_{q, a, \lambda}^{s} f(z)\right)}{D_{q, a, \lambda}^{s} f(z)}+[\lambda]\right\} \\
& =\frac{1}{[1+\lambda]}\left\{q^{\lambda} q(z)+[\lambda]\right\} .
\end{aligned}
$$

Applying logarithmic q-differentiation, we have:

$$
\frac{z d_{q}\left(D_{q, a, \lambda+1}^{s} f(z)\right)}{D_{q, a, \lambda+1}^{s} f(z)}=q(z)+\frac{z d_{q} q(z)}{q(z)+q^{-\lambda}[\lambda]}
$$

From (17) and (18), we have:

$$
q(z)+\frac{z\left[d_{q} q(z)\right]}{q(z)+q^{-\lambda}[\lambda]} \prec \frac{1+\{c(1+m)-m\} z}{1-m z} .
$$

If $\operatorname{Re}(h(z))>\frac{1-q^{-\lambda}}{1-q}$ for any value of $\lambda>-1$, so by Lemma 1 , we have $q(z) \prec h(z)$, which implies $f(z) \in S_{q, a, s, \lambda}(c, M)$. Therefore, $S_{q, a, s, \lambda+1}(c, M) \subset S_{q, a, s, \lambda}(c, M)$.

Remark 3. If we consider $q \rightarrow 1^{-}$with $\operatorname{Re} a \geq 0, c=1, m=1$ in Theorem 5 and $\lambda \geq 0, c=1, m=1$ in Theorem 7, we obtain the special cases of the inclusion results, Theorems 2.4 and 2.5 in [19].

In [30], the $q$-Bernardi integral operator $L_{b} f(z)$ is defined as:

$$
\begin{aligned}
L_{b} f(z) & =\frac{[1+b]}{z^{b}} \int_{0}^{z} t^{b-1} f(t) d_{q} t \\
& =z+\sum_{k=2}^{\infty}\left(\frac{[1+b]}{[k+b]}\right) a_{k} z^{k}, b=1,2,3, \ldots .
\end{aligned}
$$

Now, we apply the generalized operator $D_{q, a, \lambda}^{s}$ on $L_{b} f(z)$ as:

$$
D_{q, a, \lambda}^{s}\left(L_{b} f(z)\right)=z+\sum_{k=2}^{\infty}\left(\frac{[k+a]}{[1+a]}\right)^{s} \cdot \frac{[\lambda+1]_{k-1}}{[k-1] !}\left(\frac{[1+b]}{[k+b]}\right) a_{k} z^{k} .
$$

The identity relation of $D_{q, a, \lambda}^{s}\left(L_{b} f(z)\right)$ is given as:

$$
z d_{q}\left[D_{q, a, \lambda}^{s}\left\{L_{b} f(z)\right\}\right]=\left(\frac{[1+b]}{q^{b}}\right) D_{q, a, \lambda}^{s} f(z)-\frac{[b]}{q^{b}} D_{q, a, \lambda}^{s}\left\{L_{b} f(z)\right\} .
$$

The following theorems are the integral inclusions of the classes $S_{q, a, s, \lambda}(c, M)$ and $C_{q, a, s, \lambda}(c, M)$ with respect to the $q$-Bernardi integral operator.

Theorem 8. If $f(z) \in S_{q, a, s, \lambda}(c, M)$ then $L_{b} f(z) \in S_{q, a, s, \lambda}(c, M)$ if $\operatorname{Re}\left(\frac{1+\{c(1+m)-m\} z}{1-m z}\right)>\frac{1-q^{-b}}{1-q}$ for any complex numbers.

Proof. Let $g(z) \in S_{q, a, s, \lambda}(c, M)$, then:

$$
\frac{z d_{q}\left(D_{q, a, \lambda}^{s} g(z)\right)}{D_{q, a, \lambda}^{s} g(z)} \prec \frac{1+\{c(1+m)-m\} z}{1-m z} .
$$

Consider: 


$$
h(z)=\frac{1+\{c(1+m)-m\} z}{1-m z}
$$

and:

$$
u(z)=\frac{z d_{q}\left(D_{q, a, \lambda}^{s} L_{b} g(z)\right)}{D_{q, a, \lambda}^{s} L_{b} g(z)} .
$$

We will show:

$$
u(z) \prec h(z),
$$

which would prove $L_{b} g(z) \in S_{q, a, s, \lambda}(c, M)$. From the identity relation (19), after some calculations, we have:

$$
\frac{z d_{q}\left(D_{q, a, \lambda}^{s} L_{b} g(z)\right)}{D_{q, a, \lambda}^{s} L_{b} g(z)}=\left(\frac{[1+b]}{q^{b}}\right) \frac{D_{q, a, \lambda}^{s} g(z)}{\left(D_{q, a, \lambda}^{s} L_{b} g(z)\right)}-\frac{[b]}{q^{b}} .
$$

After some calculations, we have:

$$
\frac{D_{q, a, \lambda}^{s} g(z)}{D_{q, a, \lambda}^{s} L_{b} g(z)}=\frac{1}{[1+b]}\left[\frac{q^{b} \cdot z d_{q}\left(D_{q, a, \lambda}^{s} L_{b} g(z)\right)}{D_{q, a, \lambda}^{s} L_{b} g(z)}+[b]\right]
$$

Applying logarithmic $q$-differentiation, we have:

$$
\frac{z d_{q}\left(D_{q, a, \lambda}^{s} g(z)\right)}{D_{q, a, \lambda}^{s} g(z)}=u(z)+\frac{z\left[d_{q} u(z)\right]}{u(z)+q^{-b}[b]}
$$

From (20) and (21), we have:

$$
u(z)+\frac{z\left[d_{q} u(z)\right]}{u(z)+q^{-b}[b]} \prec \frac{1+\{c(1+m)-m\} z}{1-m z}
$$

If $\operatorname{Re}(h(z))>\frac{1-q^{-b}}{1-q}$, so by Lemma 1 , we have $u(z) \prec h(z)$, which implies $L_{b} g(z) \in$ $S_{q, a, s, \lambda}(c, M)$.

Theorem 9. If $f(z) \in C_{q, a, s, \lambda}(c, M)$, then $L_{b} f(z) \in C_{q, a, s, \lambda}(c, M)$ for any complex number s.

Proof. It is an immediate consequence of the fact $C_{q, a, s, \lambda}(c, M) \Leftrightarrow z d_{q} f \in S_{q, a, s, \lambda}(c, M)$.

Author Contributions: Conceptualization: K.I.N.; formal analysis: R.S.B. and K.I.N.; investigation: R.S.B. and K.I.N.; methodology: R.S.B. and K.I.N.; supervision: K.I.N.; validation: R.S.B.; writing, original draft: R.S.B. All authors read and agreed to the published version of the manuscript.

Funding: The authors received no funding for this research.

Conflicts of Interest: The authors declare no conflict of interest.

\section{References}

1. Einstein, A. Concerning on heuristic point of view toward the emission and transformation of light. Ann. Phys. 1905, 17, 132-148. [CrossRef]

2. Jackson, F.H. On q-definite integrals. Q. J. Pure Appl. Math. 1910, 41, 193-203.

3. Jackson, F.H. q-difference equations. Am. J. Math. 1910, 32, 305-314. [CrossRef] 
4. Srivastava, H.M. Univalent functions, fractional calculus and associated generalized hypergeometric functions. In Univalent Functions, Fractional Calculus and Their Applications; Srivastava, H.M., Owa, S., Eds.; Halsted Press: Chichester, UK; John Wiley and Sons: New York, NY, USA; Chichester, UK; Brisbane, Australia; Toronto, ON, Canada, 1989; pp. 329-354.

5. Ismail, M.E.H.; Markes, E.; Styer, D. A generalization of starlike functions. Complex Var. 1990, 14, 77-84. [CrossRef]

6. Agrawal, S.; Sahoo SK. A generalization of starlike functions of order alpha. Hokkaido Math. J. 2017, 46, 15-27. [CrossRef]

7. Purohit, S.D.; Raina, R.K. Certain subclasses of analytic functions associated with fractional $q$-calculus operator. Math. Scand. 2007, 109, 55-70. [CrossRef]

8. Sahoo, S.K.; Sharma, N.L. On a generalization of close-to-convex functions. Ann. Polon. Math. 2015, 113, 93-108. [CrossRef]

9. Srivastava, H.M. Operators of basic (or $q$-) calculus and fractional $q$-calculus and their applications in geometric function theory. Iran. J. Sci. Technol. Trans. Sci. 2020, 44, 327-344. [CrossRef]

10. Mohammad, A.; Darus, M. A generalized operator involving the $q$-hypergeometric function. Mat. Vesnik 2013, 65, 454-465.

11. Wilf, H.S. Subordinating factor sequence for convex maps of the unit circle. Proc. Am. Math. Soc. 1962, 12, 689-693. [CrossRef]

12. Ernst, T. A Comprehensive Treatment of q-Calculus; Springer: Basel, Switzerland; Heidelberg, Germany; New York, NY, USA; Dordrecht, The Netherlands; London, UK, 2012.

13. Ali, S.; Noor, K.I. Study on the $q$-analogue of a certain family of linear operators. Turkish J. Math. 2019, 43, 2707-2714.

14. Noor, K.I.; Bukhari, S.Z.H. Some subclasses of analytic and spiral-like functions of complex order involving the Srivastava-Attiya integral operator. Integtral Transforms Spec. Funct. 2010, 21, 907-916. [CrossRef]

15. Kanas, S.; Răducanu, D. Some classes of analytical functions related to conic domains. Math. Slovaca 2014, 64, 1183-1196. [CrossRef]

16. Owa, S.; Srivastava, H.M. Some applications of generalized Libera integral operator. Proc. Japan Acad. Ser. A. Math. Sci. 1986, 62, 125-128. [CrossRef]

17. Wang, Z.G.; Li, Q.G.; Jiang, Y.P. Certain subclasses of multivalent analytic functions involving generalized Srivastava-Attiya operator. Integtral Transforms Spec. Funct. 2010, 21, 221-234. [CrossRef]

18. Govindaraj, M.; Sivasubramanian, S. On a class of analytic functions related to conic domains involving q-calculus. Anal. Math. 2017, 43, 475-487. [CrossRef]

19. Al-Shaqsi, K.; Darus, M. A multiplier transformation defined by convolution involving $n$th order polylogarithm functions. Int. Math. Forum 2009, 4, 1823-1837.

20. Çetinkaya, A.; Polatoğlu, Y. $q$-Harmonic mappings for which analytic part is $q$-convex functions of complex order. Hacet. J. Math. Stat. 2018, 47, 813-820. [CrossRef]

21. Aouf, M.K.; Seoudy,T.M. Convolution properties for classes of bounded analytic functions with complex order defined by $q$-derivative. RACSAM 2019, 113, 1279-1288. [CrossRef]

22. Bukhari, S.Z.H.; Noor, K.I.; Malik, B. Some applications of generalized Srivastava-Attiya integral operator. Iran. J. Sci. Technol Trans. A Sci. 2018, 42, 2251-2257. [CrossRef]

23. Noor, K.I.; Riaz, S. Generalized $q$-starlike functions. Studia Sci. Math. Hungar. 2017, 54, 509-522. [CrossRef]

24. Noor, K.I. Some classes analytic functions associated with $q$-Ruscheweyh differential operator. Facta Univ. Ser. Math. Inform. 2018, 33, 531-538.

25. Polatoğlu, Y.; Şen, A. Some results on subclasses of Janowski -spiral like functions of complex order. Gen. Math. 2007, 15, 88-97.

26. Aouf, M.K.; Darwish, H.E.; Attiya, AA. On a class of certain analytic functions of complex order. Indian J. Pure Appl. Math. 2001, 32, 1443-1452.

27. Shamsan, H.; Latha, S. On generalized bounded Mocanu variation related to $q$-derivative and conic regions. Ann. Pure Appl. Math. 2018, 17, 67-83. [CrossRef]

28. Răducanu, D.; Srivastava, H.M. A new class of analytic functions defined by means of a convolution operator involving the Hurwitz-Lerch Zeta function. Integtral Transforms Spec. Funct. 2007, 18, 933-943. [CrossRef] 
29. Güney, H.O.; Attiya, A.A. A subordination result with Salagean-type certain analytic functions of complex order. Bull. Belg. Math. Soc. 2011, 18, 253-258. [CrossRef]

30. Noor, K.I.; Riaz, S.; Noor, M.A. On q-Bernardi linear operator. TMWS J. Pure Appl. Math. 2017, 8, 3-11.

(C) 2020 by the authors. Licensee MDPI, Basel, Switzerland. This article is an open access article distributed under the terms and conditions of the Creative Commons Attribution (CC BY) license (http://creativecommons.org/licenses/by/4.0/). 